\title{
La teoría de John Rawls en la jurisprudencia constitucional ${ }^{\star}$
}

\author{
John Rawls's Theory in Constitutional Jurisprudence
}

\section{A teoria de John Rawls na jurisprudência constitucional}

\section{RONALD ZULEYMAN RICO SANDOVAL}

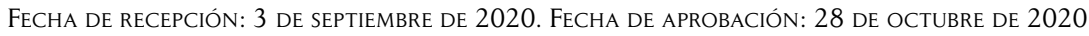

Doi: https://doi.org/10.12804/revistas.urosario.edu.co/sociojuridicos/a.9837

Para citar este artículo: Rico Sandoval, R. Z. (2021). La teoría de John Rawls en la jurisprudencia constitucional. Revista de Estudios Socio-Jurídicos, 23(1), 133-161. https://doi.org/10.12804/revistas.urosario.edu.co/sociojuridicos/a.9837

\section{RESUMEN}

En este texto pretendo exponer algunas de las ideas centrales de la Teoría de la justicia del filósofo norteamericano John Rawls. En primer lugar, examinaré las características generales de los dos principios de la justicia; en segundo lugar, analizaré cómo dichos principios se desarrollan en un Estado democrático en lo que Rawls denomina la "secuencia de las cuatro etapas". Expuesto lo anterior me concentraré en dos de esas cuatro etapas: por un lado, relacionaré la segunda de las cuatro etapas con la teoría del poder constituyente, concepto central en el constitucionalismo moderno. Por otro lado, estudiaré la última etapa de esa secuencia de cara al papel del juez en Colombia, en concreto con relación al control constitucional. Por último, intentaré mostrar cómo ha sido la recepción del autor norteamericano en la jurisprudencia de la Corte Constitucional colombiana.

Palabras clave: teoría de la justicia; posición original; poder constituyente (constitucionalismo popular); juez constitucional; corte constitucional; Rawls.

* El presente ensayo se escribió en el año 2020, dentro del programa de Asistencia de Investigación del Doctorado en Filosofía de la Pontificia Universidad Javeriana. Tiene como punto de partida el seminario doctoral sobre la Teoría de la justicia de John Rawls.

** Abogado de la Pontificia Universidad Javeriana, especialista en Derecho Constitucional de la Universidad del Rosario, Magíster en Derecho de la Universidad Sergio Arboleda, Magíster en Filosofía de la Pontificia Universidad Javeriana y becario del Doctorado en Filosofía de la misma universidad de donde es miembro del Grupo de Investigación Pensamiento Crítico y Subjetividad. Juez Civil de Bogotá, Colombia. Correo electrónico: ricor@javeriana.edu.co. ORCID: https://orcid.org/0000-0003-0747-9846 


\section{ABSTRACT}

This text tries to expose some of the central ideas of the Theory of Justice of the North American philosopher John Rawls. First, I will examine the general characteristics of the two principles of justice; secondly, I will analyze how these principles are developed in a democratic state in what Rawls calls the "four-stage sequence". Having exposed the above, I will concentrate on two of those four stages: on the one hand, I will relate the second of the four stages to the theory of popular constitutionalism (constituent power), a central concept in modern constitutionalism. On the other hand, I will study the last stage of this sequence facing the role of the judge in Colombia, specifically in relation to the constitutional control of law. Finally, I will try to show how the reception of the North American author has been in the jurisprudence of the Colombian Constitutional Court.

Keywords: Theory of justice; original position; popular constitutionalism; constitutional judge; constitutional court; Rawls.

\section{RESUMO}

Neste texto pretendemos expor algumas das ideias centrais da Teoria da Justiça do filósofo norte-americano John Rawls. Em primeiro lugar, examinaremos as categorias gerais dos dois princípios da justiça; em segundo lugar, analisaremos como tais princípios se desenvolvem em um Estado democrático, em que Rawls denomina de "a sequência das quatro etapas". Diante do exposto, nos concentraremos em duas dessas quatro etapas: por um lado, relacionaremos a segunda das quatro etapas com a teoria do poder constituinte, conceito central no constitucionalismo moderno. Por outo lado, estudaremos a última etapa dessa sequência a respeito do papel do juiz na Colômbia, especificamente em relação ao controle constitucional. Por fim, tentaremos mostrar como o autor norte-americano foi recebido na jurisprudência do Tribunal Constitucional colombiano.

Palavras-chave: teoria da justiça; posição original; poder constituinte (constitucionalismo popular); juiz constitucional; tribunal constitucional; Rawls. 


\section{Introducción}

La pregunta por el origen del Estado está a la base del derecho constitucional: ¿Por qué ha de existir un Estado? ¿Cuál es el fundamento político del poder? ¿Por qué debemos obedecer a un gobernante? Estas son preguntas que la filosofía política y del derecho intentan responder. Es en este contexto teórico que surge la llamada teoría contractualista que explica la formación estatal e, incluso, el origen de las constituciones en un acto genitor de asociación humana. Así, por ejemplo, en el capítulo XVI de su Tratado teológico-político, Spinoza (2008), tras reconocer que el ser humano nace ignorante de las leyes de la razón y se rige más bien por el deseo, proclama la necesidad de vivir en comunidad para limitar ese poder irracional del apetito. Para lograr este objetivo hombres y mujeres tuvieron que unir sus esfuerzos para prodigarse ayuda mutua:

Hicieron, pues, que el derecho a todas las cosas, que cada uno tenía por naturaleza, lo poseyeran todos colectivamente y que en adelante ya no estuviera determinado según la fuerza y el apetito de cada individuo, sino según el poder y la voluntad de todos a la vez (...) Por eso debieron establecer, con la máxima firmeza y mediante un pacto, dirigirlo todo por el solo dictamen de la razón (Spinoza, 2008, pp. 337-338).

En este punto, Spinoza parece estar muy cerca de Hobbes quien es más conocido en el ámbito jurídico. Hobbes sostiene la existencia de un estado de naturaleza previo a toda comunidad, estado que se caracteriza por el miedo o temor mutuo de los diversos individuos. Existe, entonces, una razón egoísta que explica la existencia del Estado, esto es, la necesidad de asegurar la vida y la propiedad (Hegel, 1979). Hegel (1979) concluye que la característica de los individuos en ese estado de naturaleza "no se basa, como en los tiempos modernos, en la libertad del espíritu o en la dignidad y la autonomía de los hombres, sino en la igual debilidad de todos ellos" (p. 333).

En el caso de Spinoza (2008), el pacto colectivo impone una asociación que se rige por la razón, mientras que en Hobbes lo que se impone es un poder soberano que mediante el ejercicio de la fuerza 
somete a los individuos. Sobre esto último, Walter Benjamin (2010), en su bien conocido ensayo: Hacia la crítica de la violencia, señala que en la base del poder - Gewalt, como lo interpreta Derrida (2010) - se encuentra el ejercicio de la violencia que instaura el derecho (del Estado) y la que lo conserva; es decir, que el poder estatal se sustenta en un acto de violencia permanente. No en vano, "para Hobbes el estado de naturaleza no es un estado imaginario, sino una amenaza siempre presente" (Jaramillo et al., 2018, p. 183) y que por tal debe ser controlada por ese soberano.

Estas dos percepciones sobre el estado de naturaleza, propias del siglo XVII, han marcado la visión que se tiene del llamado contrato social. Piénsese que en ambos casos el Estado se sustenta en la necesidad de limitar el deseo o la violencia de los hombres, que solo difieren en la forma en que ha de darse dicha limitación: en un caso, la razón de la multitud y, en el otro, la voluntad del soberano.

Autores como Locke sostienen un contractualismo orientado a la protección de la vida y la propiedad privada basados en un derecho natural, pero que, con todo, reconocen la inseguridad de tales derechos en un estado no asociativo o estado de naturaleza que se caracteriza por el uso de la fuerza (Cfr. Jaramillo et al., 2018). De igual manera, Kant (2008) reconoce como hipótesis racional que el estado previo a la conformación de un Estado legal público es la de "hombres, pueblos y Estados aislados [que] nunca pueden estar seguros unos de otros frente a la violencia[; por ende, lo primero que debe hacer el hombre es] salir del estado de naturaleza, en el que cada uno obra a su antojo, y unirse con todos los demás (...) para someterse a una coacción externa legalmente pública" (§44, p. 141).

Las teorías contractualistas tuvieron, si se quiere, un gran apogeo como fuente de la que se nutrió la Revolución Francesa y el proceso constitucional que le siguió, esto por cuenta de la influencia de la llamada voluntad general expuesta en El contrato social de Rousseau y de la teoría de la representación de esa voluntad ideada por el abate Sieyès (Cfr. Jaramillo et al., p. 546). Allí se expresaba el fundamento del Estado y el establecimiento de instituciones políticas que lo desarrollaban.

No obstante, instauradas las repúblicas en Occidente pareciera que la teoría del contrato social pasó a convertirse en una mera anécdota 
de libros de derecho constitucional las más de las veces destinados a explicar antecedentes de las constituciones vigentes o para justificar el voto y la representación legislativa. Así fue, al menos en lo que respecta a la bibliografía nacional, por ejemplo, en las obras de Cerbeleón Pinzón (Tratado de ciencia constitucional); Antonio del Real (Elementos de derecho constitucional); José María Samper (Derecho público interno); José Vicente Concha (Apuntamientos de derecho constitucional), Francisco de Paula Pérez (Estudios constitucionales), Tulio Enrique Tascón (Derecho constitucional colombiano), por mencionar algunos autores colombianos del siglo XIX y de comienzos del XX (Cfr. Rico, 2005).

Se puede afirmar, entonces, que el contractualismo como tesis filosófico-jurídica había perdido importancia. En el ámbito anglosajón, el panorama no parecía ser muy diferente; Leonardo García Jaramillo (2004, pp. 97-ss.) explica que a mediados del siglo XX, la filosofía política estaba tan desprestigiada que se la entendió como "muerta". Los debates políticos, jurídicos y filosóficos se reducían a problemas de gobernabilidad y validez electoral. Ese era el contexto del contractualismo cuando se publicó, en 1971, la Teoría de la justicia de John Rawls.

Dicha obra marcó un hito en la justificación racional de la justicia y significó un renacer de la filosofía política y, en concreto, de la teoría contractualista. Esto es así porque Rawls (2011) supo insistir en la importancia de la tradición de autores como John Locke e Inmanuel Kant, entre otros (Nussbaum, 2004). Dicho texto no solo resulta de especial importancia para la filosofía política contemporánea, sino que ofrece especiales aportes para la teoría constitucional actual. En efecto, a partir de Rawls, la filosofía política resurge con una teoría interesante: es posible elegir, de manera racional, unos principios de la justicia que deberán estructurar todo Estado bien organizado. ${ }^{1}$

La tesis puede resumirse de la siguiente manera: es válido pensar filosóficamente un pacto social donde las personas elijan, de manera racional, los principios básicos para la formación de un Estado justo, los cuales deberán quedar plasmados en una constitución que, a la vez, consagre instituciones fuertes que garanticen una convivencia justa en

1 Rawls escribe sobre una sociedad bien organizada. Dado que este ensayo se dirige a analizar la formación de un Estado justo, acá se utilizará la expresión Estado bien organizado. 
sociedad. Por esto, sus intérpretes consideran que "Rawls se ocupó de la organización política de una determinada sociedad, de lo que es su institucionalidad general, de lo que la articula como una cooperación estable entre diferentes partes unidas por un mismo vínculo cívico" (Grueso, 2005, pp. 15-16).

El ejercicio teórico que formula Rawls (2011) parte de considerar una hipótesis lógica similar al estado de naturaleza expuesto por diferentes filósofos antes que él. Sin embargo, dicha conjetura no se apoya en una teoría antropológica que considere a los humanos como seres salvajes por naturaleza, que necesiten pasar a un estadio asociativo para restringir la violencia. Su teoría es más interesante: formula un estadio neutro, pero racional, al que llama "posición original", en el que los sujetos pueden escoger unos principios de la justicia que sean favorables a todos en la medida que sobre ellos pesa un "velo de ignorancia" que les impide conocer su posición en la sociedad, por ende, no podrían establecer privilegios para unos en detrimento de otros.

El objetivo principal de Rawls es la formulación de los principios de la justicia, por lo que su teoría contractualista tan solo sería una premisa metodológica para explicar la elección de esos principios. Sin embargo, para el mantenimiento de esos principios de la justicia se requiere de un andamiaje estatal y es desde esa perspectiva que la teoría rawlsiana resulta interesante desde el punto de vista constitucional, de cara a dos conceptos claves: la figura del poder constituyente y el establecimiento de instituciones estatales.

En este artículo se quiere relacionar la teoría del filósofo norteamericano con la figura del poder constituyente primario, para lo cual se seguirá el siguiente orden: en primer lugar, se expondrá cómo funciona la teoría rawlsiana con miras a la formación de un Estado; en segundo lugar, se relacionará dicha teoría con la génesis del Estado según la teoría del poder constituyente primario; en tercer lugar, se destacará el papel del juez constitucional en un Estado bien ordenado, para arribar a cómo se ha introducido a Rawls en la jurisprudencia constitucional colombiana. 


\section{La teoría de la justicia de John Rawls}

En este apartado se expondrán los elementos característicos de la teoría rawlsiana de la justicia tal y como fueron expuestos en Teoría de la justicia. Sin embargo, por la brevedad de este texto solo se abordarán los siguientes: la estructura básica, el velo de ignorancia, la posición original y los principios de justicia. Por consiguiente, no se analizarán los conceptos de equilibrio reflexivo, consenso traslapado ni orden lexicográfico de los principios, entre otros. En el siguiente apartado se estudiará cómo opera el velo de ignorancia de cara a una convención constituyente.

\section{La estructura básica}

En su libro: Teoría de la justicia, Rawls se ocupó de la justicia en un Estado bien ordenado. Para ello plantea que si se desea un Estado justo, este debe apoyarse en unos principios elementales que este mismo formula y delimita. Para demostrar que son esos los principios que deben inspirar a un Estado, el filósofo norteamericano establece un procedimiento que parte de una hipótesis teórica que denomina "posición original" que garantiza unas condiciones de imparcialidad. Las personas, que a la postre integrarán un Estado, deberán elegir los principios basilares de la justicia que de momento llamaremos los principios que garantizan las libertades y las igualdades. Una vez elegidos dichos principios, su existencia se debe garantizar mediante unas instituciones estatales fuertes, las que se consagran en una constitución política. Desde otra óptica, se puede afirmar que una vez se elijan los principios que definen y gobiernan la justicia, estos servirán de pauta para evaluar la forma como un Estado distribuye derechos y cargas. En esto consiste la llamada estructura básica, esto es, "en la creación de instituciones justas" (Puyol, 2016, p.7), que garanticen los principios de la justicia. Dice Rawls (2011):

digamos que una sociedad está bien ordenada no solo cuando fue organizada para promover el bien de sus miembros, sino cuando también está eficazmente regulada por una concepción pública de la 
justicia. Esto quiere decir que se trata de una sociedad en la que: 1) cada cual acepta y sabe que los demás aceptan los mismos principios de justicia, y 2) las instituciones sociales básicas satisfacen generalmente estos principios y se sabe generalmente que lo hacen (p. 18).

En otro texto, Liberalismo politico, Rawls (2015) resume su concepto de estructura básica como

la manera en que las más importantes instituciones sociales encajan unas en otras en un sistema, y en cómo asignan derechos y deberes fundamentales y dan forma a la división de las ventajas que se obtienen mediante la cooperación social (p. 188).

Rawls, entonces, parte de dos supuestos. Por un lado, piensa que una sociedad es una agrupación de personas que actúan según un conjunto de reglas que garantizan un sistema de cooperación, cuyo fin es promover el bien de sus integrantes. Esta asociación cooperativa hace posible una vida mejor que la que se pudiera tener si se viviera en estado de soledad. Pero, como en toda sociedad, en ella existiría un conflicto entre los asociados por la distribución de los mayores beneficios que tal empresa generara o sobre las cargas que esta demande. Por otro lado, considera que, bajo ciertas condiciones, los integrantes de dicha sociedad elegirían los principios de la justicia, de manera objetiva y racional.

Ahora bien, para que sea posible un acuerdo fundamental sobre los principios que gobernarán la sociedad, Rawls acude a la teoría de la elección racional. En primer lugar, parte de la identificación de un sujeto que puede hacer la elección, esto es, un sujeto racional. En efecto, en clara alusión a Kant, Rawls considera que la partes contratantes de ese contrato social son "personas morales libres e iguales" (Rawls, 2015, pp. 188-189).

En segundo lugar, Rawls esboza la hipótesis contractualista clásica, esto es, que las personas se pueden ubicar en una posición primigenia, anterior al Estado, para, de manera racional, efectuar su elección. Esa hipotética ubicación es denominada la "posición original", un estadio donde los ciudadanos debatirían sobre cuáles serían los principios de una sociedad bien ordenada. 


\section{El velo de ignorancia y la posición original}

Para que esta ficción funcione, el filósofo norteamericano plantea otro concepto básico de su teoría, el llamado "velo de ignorancia": un velo que cubriría a todas las personas en esa posición original a efectos de que ellas no conozcan esos datos particulares que les otorgarían ventaja al momento de determinar los principios de la justicia de un Estado. En esta posición, los ciudadanos deben hacer caso omiso de las contingencias; es decir, deben actuar sin saber cuál sería el sexo, la raza, la posición social y económica que le correspondería a cada cual en esa futura sociedad bien organizada. La razón de ser de esta premisa es que nadie puede estar ubicado "en una posición ventajosa o desventajosa por la fortuna natural o por las circunstancias sociales al escoger los principios" (Rawls, 2011, p. 30).

Sin embargo, en tal posición y con ese velo hipotético no se tendría sujetos totalmente vaciados de contenido, ya que esos ciudadanos libres y morales deberían acceder a un mínimo de conocimiento contextual de la sociedad para poder identificar los principios comunes según un contexto histórico. El filósofo del derecho norteamericano Ronald Dworkin (2014) explicó cómo funcionaría ese velo:²

para fijar principios de justicia destinados a la estructura básica de nuestra comunidad política, debíamos imaginar a personas que se reúnen para establecer dicha comunidad y están bien al corriente de los hechos económicos, tecnológicos, psicológicos y sociológicos generales, pero ignoran su propia edad, su género, sus talentos, su posición social y económica, sus intereses, deseos y creencias éticas sobre cómo vivir bien (p. 79).

No podemos olvidar, como insistentemente lo señaló Rawls (2015), que la posición original "representa el resultado de un proceso racional de deliberación en condiciones ideales y no históricas" (p. 199), por eso, la apuesta rawlsiana se dirige a plantear las condiciones de imparcialidad

2 La explicación que provee Dworkin es dentro del marco de la crítica que realiza a algunos aspectos de la teoría rawlsiana. Con todo, creemos que resulta pertinente su descripción. 
necesarias para la elección racional de los principios de la justicia para una sociedad bien organizada. Esta teorización, sin embargo, no ha estado exenta de críticas, como bien lo destaca Thomas Nagel (2003), quien comenta la debilidad de la posición original, y por ende del velo de ignorancia, porque supone que una persona "sería racional al elegir como si su enemigo fuera a asignarle su lugar en la sociedad resultante" (p. 33). No obstante, Nagel (2003) destaca que el fundamento de la teoría de Rawls debería ser "la prioridad dada a las libertades básicas, la igualdad política y legal, unas condiciones materiales decentes para la vida, y las bases del auto-respeto" (p. 33), que constituyen el núcleo de los principios de la justicia, que formula en contraposición al estándar utilitarista que maximiza las expectativas promedio, afectando con ello a los menos afortunados.

\section{Los principios de la justicia}

Estas condiciones de imparcialidad en las que se sustenta el velo de ignorancia, implican que los ciudadanos libres y morales han de saber ciertos aspectos de la sociedad que quieren ordenar, pero no deben saber datos que los beneficien a ellos directamente. Señala Rawls (2011) que si las personas en la posición originaria no pueden obtener ventajas especiales para sí mismos ni querer desventajas, "lo más sensato es reconocer como primer paso un principio de justicia que exija una distribución igualitaria" (pp. 147-148). Esto significa que las partes en dicha posición comienzan con iguales libertades básicas y así mismo deben esperar una distribución equitativa de ingresos, riquezas y oportunidades. Empero, el filósofo norteamericano reconoce que "la sociedad debe tomar en cuenta la eficiencia económica y las exigencias de la organización y de la tecnología" (Rawls, 2011, p. 148), motivo por el cual no parten de una posición de solo ignorancia. Solo en esa condición, se elegirían los principios de la justicia que serían, para Rawls, solo dos: la libertad y la igualdad. Dworkin (2014) resume preliminarmente los principios así:

Rawls sostenía que en esa extraña situación la gente coincidiría en dos principios de justicia: uno que asigna prioridad a determinadas libertades y otro que exige una estructura económica en la cual la 
situación del grupo en peores condiciones económicas es tan buena como puede permitirlo una estructura básica (p. 79).

El primer principio expuesto en su Teoría de la justicia fue reformulado en un texto posterior que agrupa varias conferencias de Rawls y que se llama Liberalismo político. Allí, el filósofo norteamericano afirma que "cada persona tiene igual derecho a un esquema plenamente adecuado de libertades básicas iguales que sea compatible con un esquema semejante de libertades para todos" (Rawls, 2012, p. 271). Lo anterior quiere decir que la garantía de la libertad como principio básico de un Estado bien ordenado se logra atribuyendo a todas las personas un esquema amplio de libertades básicas. En Liberalismo político el listado de libertades básicas se presentó así:

libertad de pensamiento y libertad de conciencia; las libertades políticas y la libertad de asociación, así como las libertades que especifican la libertad y la integridad de la persona; $y$, finalmente, los derechos y libertades que protegen las reglas de la ley (Rawls, 2015, p. 211).

Debemos aclarar que en la versión original de Teoría de la justicia, Rawls incluía la propiedad privada como libertad básica, pero en el segundo ya la trata como un presupuesto para el desarrollo de las demás libertades básicas, aunque igual de importante a estas.

El segundo principio, está asociado a las desigualdades. Originalmente fue formulado, en Teoría de la justicia, de la siguiente manera: "Las desigualdades sociales y económicas habrán de ser conformadas de modo tal que a la vez que: a) se espere razonablemente que sean ventajosas para todos, b) se vinculen a empleos y cargos asequibles para todos" (Rawls, 2011, p. 68).

Por la redacción un poco compleja, el segundo principio se presenta con un doble contenido, por un lado, lo que se denomina el "principio de igualdad de oportunidades" $y$, por el otro, el "principio de la diferencia". El primero, plantea que todas las personas deben estar ubicadas, respecto de los demás, en una situación de igualdad de tal manera que las desigualdades sociales y económicas no se distribuyan arbitrariamente, sino en función de situaciones ventajosas para todos (no para una 
mayoría como en el utilitarismo), lo que se lograría permitiendo que todos tengan una justa igualdad de posibilidades de acceso a empleos. Esto último, a su vez, requiere una educación igualitaria para todos, ya que, sin importar si se es rico o pobre, dos personas con la misma preparación tendrían, en teoría, la misma oportunidad de acceder a un empleo.

La segunda cara del principio de igualdad, esto es, el llamado "principio de diferencia", apunta a que "las ventajas de los más favorecidos solo serán aceptadas si a su vez benefician a todos, con especial énfasis en el hombre menos aventajado" (Martínez, 2015, p. 104). Dicha regla se expresa así: "las expectativas más elevadas de quienes están mejor situados son justas si y solo si funcionan como parte de un esquema que mejora las expectativas de los miembros menos favorecidos de la sociedad" (Rawls, 2011, pp. 80-81). Tras analizar la situación de los menos favorecidos, Rawls (2011) llega a la siguiente reformulación de ese segundo principio:

Las desigualdades sociales y económicas habrán de disponerse de tal modo que sean tanto a) para el mayor beneficio de los menos aventajados, como b) ligadas con cargos y posiciones asequibles a todos en condiciones de justa igualdad de oportunidades (p. 88).

Vemos que la enunciación original del segundo principio apunta a una distribución de las desigualdades sociales y económicas en busca de una posición ventajosa para todos, en tanto que la segunda formulación plantea la necesidad de que dicha distribución se haga en función del mayor beneficio de los menos aventajados. Esta perspectiva apunta a establecer un deber de justicia, "cuyos beneficios podría exigir el pobre como un derecho [porque su aplicación es] una cuestión de justicia puramente procesal" (Freeman, 2016, pp. 95 y 97).

Los "menos aventajados" que menciona Rawls vendrían a ser aquellas personas que no pueden acceder, por igual, a los llamados "bienes primarios, esto es, cosas que se presume que todo ser racional desea" y que el filósofo resume como "derechos, libertades, oportunidades, ingreso y riqueza" (Rawls, 2011, p. 69). La distribución de tales bienes primarios sería el punto de partida para analizar si hay una situación de 
injusticia y, por ende, para justificar la elección del segundo principio de justicia. ${ }^{3}$

Según Rawls (2011), ese deber de justicia ha de generar una "justa igualdad de oportunidades", pero no entendido de manera literal, sino solo en oposición al establecimiento de beneficios en razón a cuestiones hereditarias o de estatus socioeconómico. En este sentido, "suponiendo que existe una distribución de las capacidades naturales, aquellos que están en el mismo nivel de capacidades y habilidades y tienen la misma disposición para usarlas, deberían tener las mismas perspectivas de éxito" (Rawls, 2011, p. 78).

Este acceso igual a oportunidades no se logra ofreciendo el mismo empleo a varias personas en situaciones de desigualdad, ya que resulta lógico intuir que quien esté mejor preparado pueda acceder a mejores cargos. Es por ello que Rawls apuesta por un igual acceso a la educación para que cada cual pueda desarrollar su propio proyecto de vida; "la justa igualdad de oportunidades impone a la sociedad un deber positivo de ofrecer oportunidades educativas para que quienes tengan talentos similares, pero en desventaja social, puedan competir en términos equitativos con los más favorecidos por su clase social" (Freeman, 2016, pp. 98-99).

Hasta aquí se ha expuesto cómo los ciudadanos elegirían racionalmente los principios que estructuran la justicia de un Estado bien ordenado. En el siguiente acápite explicaremos cómo se materializan esos principios de la justicia en un acuerdo constitucional.

\section{Rawls, la convención constituyente y la estructura del Estado}

En este apartado se explicarán las cuatro fases del llamado descorrimiento del velo de ignorancia y ubicaremos en dicho proceso a la figura jurídica del poder constituyente.

3 Para Rawls los principios de justicia no están propuestos de manera arbitraria de tal manera que cada cual eligiera, según una situación determinada, aplicar uno u otro. Es por esto que establece un orden de los principios, que denomina lexicográfico. La idea es que el segundo principio está subordinado al primero y, por ende, para el filósofo resulta importante partir de los bienes primarios. 


\section{De la posición original a la convención constituyente}

Algunos autores tienden a criticar la teoría de Rawls porque consideran que la posición original es un imposible fáctico en la medida en que "ni en el mejor, ideal y más cortés de los mundos se podría encontrar una asamblea de ciudadanos dispuestos a pasar por alto tan desinteresadamente sus fines particulares" (Mancha, 2009, pp. 272-273). Esta posición parte de la premisa según la cual solo una asamblea de ciudadanos puede hacer parte en el "negocio fundamental" que da existencia a una comunidad política.

Por lo anterior, se debe aclarar la naturaleza de la posición original para no confundirla con la convención constituyente. Para ello se insistirá en tres aspectos: primero, que la posición original es una situación hipotética, lo cual quiere decir que no existió como un evento histórico identificable.

Así pues -señala Rawls-, resulta evidente que el contrato social debe considerarse hipotético y no histórico. La explicación de esto reside en que el acuerdo, en la posición original, representa el resultado de un proceso racional de deliberación en condiciones ideales y no históricas (Rawls, 2015, p. 199).

Segundo, que esa hipótesis tiene por finalidad explicar la necesidad de los principios de la justicia. Según esta premisa, los ciudadanos libres y morales elegirían unos principios comunes que permitirían estructurar un Estado justo. Tercero, solo cuando esas personas imaginadas, los ciudadanos libres y morales, han escogido esos principios de la justicia, es cuando ciudadanos concretos pueden reunirse en una asamblea $o$ convención constituyente para crear, ahora sí, un Estado y darle su propia constitución.

Entonces, la "posición original" es una situación hipotética de imparcialidad donde los ciudadanos se despojan de sus intereses particulares para elegir los principios de la justicia, bajo la premisa de que el primero desarrolla las llamadas libertades básicas distribuidas por igual en los miembros de la sociedad y el segundo atiende a la distribución de ventajas y cargas en relación con unos bienes básicos, procurando 
la mejoría de las personas menos aventajadas - suele describirse como principio de diferencia-. Esa situación de despojo de intereses particulares es lo que Rawls denomina el "velo de ignorancia". Ese velo debe cubrir, metafóricamente, los ojos de los ciudadanos de tal manera que su visión de las condiciones particulares de la sociedad sea limitada y por tal razón, en la posición original, sería "espeso", como afirma Rawls (2015, p. 198). En este sentido, si el velo es considerado "espeso" en la posición original, cuando ya no se esté ubicado allí el velo sería delgado. Para lograr la creación de un Estado bien ordenado, Rawls propone que los participantes ubicados en la posición original deberán quitarse el velo de ignorancia que les impide ver las cuestiones particulares de cada individuo en la sociedad, lo cual ocurre en cuatro etapas: a) las personas se ubican en la posición original donde los participantes no conocen sus características particulares, solo conocen unos aspectos generales de la sociedad, aquí el velo es más espeso; b) las personas se agrupan en un congreso o asamblea constituyente donde ya pueden conocer datos de su sociedad, aunque desde cierta generalidad, aquí el velo sería menos espeso; c) la sociedad instituye un cuerpo legislativo encargado de confeccionar leyes justas, de manera general, pero con base en debates políticos y económicos concretos, aquí el velo sería más delgado, y d) que William A. Edmundson llama residual (2017, p. 65), donde ya no habría velo, sería la etapa de la aplicación de las reglas a casos particulares, donde se destacan las figuras del juez y los administradores como parte de las instituciones del Estado. Por esto, Edmundson (2017) también la llama "la etapa administrativa/judicial porque comprende la revisión judicial de la legislación para asegurar su adecuación a la constitución, así como la ejecución de la legislación y el comportamiento de las personas en su papel de ciudadanos" ${ }^{\prime \prime}$ (p. 65). En este texto no se hará referencia al papel de los ciudadanos, sino solo a la estructura estatal.

La primera etapa ya quedó expuesta. Ahora se continuará con las siguientes etapas. Sobre la segunda, esto es, la del congreso o asamblea constituyente, Rawls (2011) afirma:

4 Traducción propia. 
supongo que, una vez que las partes han adoptado los principios de justicia en la posición original, procederán a efectuar un congreso constituyente. Habrán de decidir allí acerca de la justicia de las formas políticas y escoger una constitución: son los delegados, por así decirlo, ante tal congreso. Habrán de planear, dentro de los límites de los principios de justicia previamente seleccionados, un sistema relativo a los poderes constitucionales del gobierno, así como los derechos fundamentales de los ciudadanos. Es en esta etapa donde deberán ponderar la justicia de los procedimientos que resolverán las controversias políticas. Dado que se ha convenido ya en la concepción de la justicia, el velo de la ignorancia se verá parcialmente levantado (p. 188).

De esta manera, la teoría contractualista de Rawls no se queda en el mero acuerdo de la posición original donde las personas llegan a un "equilibrio reflexivo" sobre cuáles han de ser los principios de la justicia, el diseño que propone el filósofo norteamericano se extiende hasta la creación misma del Estado mediante un acuerdo constitucional. En la constitución de un Estado se materializan los acuerdos sociales que versan sobre las instituciones estatales (estructura del Estado) y los derechos de las personas. Todo el Estado y la plataforma de derechos deben estar en consonancia con los principios de justicia. En este punto creemos importante destacar que no resulta suficiente el que en la constitución se reconozcan los principios antes enunciados, así como las libertades básicas y los bienes básicos o primarios. Será necesario, además, que existan instituciones que garanticen esos principios, libertades y bienes básicos.

En esta segunda etapa, el congreso constituyente debe elaborar una "constitución justa" a partir de reglas generales mediante la incorporación de los principios de justicia como criterio desde el cual se estructura el Estado. Para asegurar un Estado bien ordenado, donde primen los principios de la justicia, se requiere una legislación justa y un sistema de protección de dichos principios. Es decir, el desarrollo de esas reglas constitucionales generales se realizaría en las dos siguientes etapas, mediante legislación y administración y decisión judicial. Esta sería una síntesis de algunas ideas expuestas en las dos primeras partes de Teoría de la justicia de Rawls, pero esto debe ser complementado con la tercera parte donde se expone una teoría del bien; empero, aquí no 
se abordará este punto, pues el objetivo es analizar las instituciones, y en concreto la función judicial.

Para Rawls (2011), los dos principios de justicia deben quedar plasmados en la constitución; sin embargo, como existe una cierta prelación del primero sobre el segundo, ${ }^{5}$ se dice que el primer principio tiene mayor importancia en la etapa constituyente en tanto que el segundo tendrá mayor relevancia en la etapa legislativa. Lo anterior, obedece a que en la constitución deben quedar consagradas "las libertades fundamentales de la persona y las libertades de conciencia y pensamiento" (p. 190), en tanto que en la elaboración de leyes justas el principio de diferencia interviene en el diseño de políticas económicas y sociales concretas.

Para Rawls (2011), el segundo principio prescribe que las políticas sociales y económicas deben asegurar el cumplimiento de las expectativas a largo plazo de los menos aventajados y ello implica un conocimiento concreto de la situación de un Estado (por ello se está en la tercera etapa del descubrimiento del velo de ignorancia) y la consagración de mecanismos concretos mediante el desarrollo legislativo.

Hasta aquí se entendería que lo político de la teoría de la justicia estaría relacionado con la fundación del Estado, la convención constituyente y el cuerpo legislativo, ya que dichos escenarios son deliberativos y permitirían proteger y materializar los principios de justicia. La cuarta y última etapa de la secuencia para eliminar el velo de ignorancia se refiere a la interpretación y aplicación de reglas jurídicas y corresponde, principalmente, a jueces y administradores, quienes deben conocer las características y circunstancias de los casos concretos. Si bien algunas de las consideraciones del siguiente apartado podrían predicarse de la administración del Estado, tan solo se hará referencia al papel del juez.

\section{El poder constituyente}

En el campo del derecho, el surgimiento del Estado se ha sustentado en "la voluntad de los miembros de la comunidad política: [ya que] la

Dicha prelación es a la que anteriormente se aludió como "orden lexicográfico" y significa que para poder considerar el segundo principio de la justicia se debe primero satisfacer el primero. Es decir, la libertad es el presupuesto básico para que se pueda hablar de distribución justa de las cargas y ventajas en un Estado. 
constitución se concibe como la realización práctica del pacto social" (Jaramillo et al., 2018, p. 128). En efecto, en Colombia, de tiempo atrás, se suele estudiar o enseñar el llamado contrato social como parte del origen de la formación del Estado. Ahora bien, dicha teoría contractualista, que en el campo del derecho ha tenido diversas interpretaciones, en la actualidad se expresa bajo la premisa del poder que tiene el ente abstracto llamado "pueblo" para darse una propia constitución. Este poder se denomina "poder constituyente" y se le asigna el calificativo de "primario" para diferenciarlo del poder "secundario", que reside en el congreso o parlamento.

Así, por ejemplo, la Corte Constitucional colombiana, ${ }^{6}$ en una sentencia hito, reiteró la importancia del concepto de poder constituyente primario, a modo de realización concreta del llamado contrato social; dijo en ese entonces la Corte:

En el mundo contemporáneo, en desarrollo de los principios democráticos y de la soberanía popular, el poder constituyente está radicado en el pueblo, quien tiene y conserva la potestad de darse una Constitución (...). Por ello, tanto esta Corte Constitucional, como la Corte Suprema de Justicia durante la vigencia de la Constitución de 1886, han sostenido invariablemente que los actos del poder constituyente originario son fundacionales, pues por medio de ellos se establece el orden jurídico, y por ello dichos actos escapan al control jurisdiccional (Sentencia C-551 de 2003).

En dicha sentencia, la Corte destacó que la teoría del poder constituyente hace alusión al poder reformador del pueblo "entendido este concepto como los ciudadanos que participan en una asamblea constituyente o en la elección de los miembros de un congreso que actúa como constituyente derivado o, incluso, al decidir sobre un referendo reformador de la constitución" (Sentencia C-551 de 2003).

La teoría del constituyente primario, entonces, consiste en una justificación política de la creación de un Estado, lo que se materializa o concreta en una constitución. Tal hipótesis, por tratarse de una teoría

6 Todas las sentencias aquí citadas fueron consultadas directamente de la página web de la Corte Constitucional. 
contractualista, encuentra eco en la formulación que realiza Rawls, según se expuso en el acápite anterior. En efecto, como señala el filósofo español Ángel Puyol González (2016), "Rawls se ciñe a la clásica concepción hobbesiana de que no hay justicia sin Estado" (p. 122) y por ello el interés por justificar racionalmente los principios de la justicia se trasforma en la necesidad de crear un Estado. Es decir, tanto la doctrina jurídica del poder constituyente como el procedimiento de cuatro etapas antes estudiado se dirigen a crear y organizar un Estado bien ordenado.

Sin embargo, debemos insistir en que la asamblea constituyente que menciona la Corte Constitucional no se equipara a la posición original, sino que aquella consiste en un estadio posterior. Es decir, en la teoría rawlsiana solo se podría llegar a dicha asamblea constituyente una vez se hayan escogido los principios de la justicia necesarios para saber cómo ha de estructurarse el Estado lo cual ocurre en la posición original.

La importancia de la etapa constitucional, explica Freeman (2016), es que en ella cada sociedad puede definir su propia constitución según su historia, cultura, recursos y nivel de desarrollo (p. 193). Pero para que la constitución que allí se genere sea la de un Estado bien ordenado y justo, se requiere la participación de todos los ciudadanos de forma libre e igual, por lo que la etapa de la convención constituyente debe encarnar alguna forma de regla democrática, lo que se ajusta teóricamente a la figura del poder constituyente primario tal y como se la ha entendido en Colombia.

Hasta aquí se ha querido presentar una articulación entre la teoría rawlsiana de la justicia y la teoría constitucional colombiana que se ejemplifica en la llamada convención constituyente. Sin embargo, se quiere dar un paso más en este análisis hacia la cuarta etapa del descorrimiento del velo de ignorancia, la cual queremos entender como la "etapa judicial" sin perjuicio de los deberes que la administración y los ciudadanos tienen según la exposición que hace Rawls (2011; 2012; 2015). 


\section{El impacto de la filosofía de Rawls en Colombia}

Rawls, en su empeño por imprimirle un perfil político a su teoría de la justicia como equidad, pareciera dejar de lado la aplicación práctica. Ello se debe a que su interés en Teoría de la justicia era "generalizar y llevar a un más alto nivel de abstracción la tradicional doctrina del contrato social" (Rawls, 2015, p. 9), como lo reconoce al comienzo de Liberalismo político. No obstante, para la teoría constitucional actual es tan importante la estructuración del Estado y la participación política ciudadana, como el papel del juez, lo que ha permitido implantar en el ámbito local teorías de cuño anglosajón sobre la actividad judicial. Queremos pensar entonces, sin desconocer el marcado carácter político de la propuesta rawlsiana, que la teoría de la justicia expuesta por Rawls tiene un nivel práctico que se concreta en lo técnico-jurídico más allá del simple establecimiento de instituciones estatales.

Este aspecto de lo concreto se materializa en el papel del juez y no tanto en el del administrador del Estado, ya que son los jueces quienes deciden sobre la interpretación y aplicación de las leyes en defensa de las libertades básicas. Esto se da no solo en la revisión judicial de las leyes para verificar que estén acordes a la Constitución, sino también cuando se analizan casos sobre derechos fundamentales.

A continuación se expone el papel del juez y en general de la justicia, en el marco de la Teoría de la justicia de Rawls, y se explica cómo ha sido la introducción de dicha teoría en la jurisprudencia de la Corte Constitucional colombiana.

\section{El papel del juez en Teoría de la justicia de Rawls}

En la teoría rawlsiana, el rol del juez se analiza dentro de la cuarta etapa de descorrimiento del velo de ignorancia. Esta última etapa se caracteriza por el llamado "imperio de la ley". Para comprender lo anterior deben tenerse en cuenta dos cosas: el concepto de ley y la ausencia de un formalismo jurídico en Rawls.

Respecto de lo primero, para Rawls (2011), las leyes definen cómo cada individuo puede realizar sus legítimas expectativas, pero también limitan el ejercicio de ciertas libertades, por ende, estas deben ser claras 
y públicas a efectos de que los ciudadanos sepan qué pueden hacer y a qué deben enfrentarse en caso de desobediencia. Además, para poder hablar de un imperio de la ley, las reglas jurídicas reclaman de una aplicación regular y no arbitraria o caprichosa. Por esto último, el filósofo norteamericano prefiere hablar de "justicia como regularidad" (Rawls, 2011, p. 222). Sobre lo segundo, se debe aclarar que el llamado imperio de la ley en Rawls no implica una justicia formal por parte de los jueces, esto es, aquella que privilegia las formas jurídicas, sino que demanda una aplicación apropiada de la ley y, además, que esta sea interpretada de forma correcta.

Por consiguiente, para que exista una administración de justicia que se adecúe a los parámetros de justicia del Estado, previamente definidos en la constitución y en la ley, se requiere: a) un sistema claro de reglas que regulen los juicios y la práctica de pruebas; b) un sistema judicial permanente no solo para resolver juicios ad hoc; c) de jueces que sean independientes e imparciales, que no puedan ser influenciados "por el clamor público"; 7 d) de la regularidad y igualdad: los casos similares deben ser tratados de forma similar, lo que implicaría un sistema de precedentes que debe ser acatado por los jueces, y e) del respeto de la discrecionalidad interpretativa que no es lo mismo que la arbitrariedad.

Sobre esto último, es pertinente aclarar que la interpretación de la ley debe respetar los parámetros que ella misma imponga, así como los principios para ello. No obstante, los jueces tienen cierta autonomía hermenéutica que les permite, por ejemplo, apartarse de casos similares anteriores siempre que justifiquen por qué debe realizarse esa distinción, o cuando adviertan que debe hacerse una excepción "porque la norma establecida produce una injusticia imprevista" (Rawls, 2011, p. 224).

Hasta aquí la caracterización del rol judicial en Teoría de la justicia, al menos de manera formal. Corresponde ahora ver cómo las anteriores características, así como la protección de los menos favorecidos (segundo

Este tema de la independencia judicial se desarrolla normativamente en el artículo 228 de la Constitución y en el artículo 5 de la Ley 270 de 1996, Estatutaria de la Administración de Justicia, pero solo como filtro que debe impedir la intromisión de fuerzas externas en la decisión judicial. Lo anterior no impide que un juez o jueza se vea influido por factores externos como la religión o la ideología, tal y como lo señala Duncan Kennedy (2010). 
principio de la justicia) han tenido cierto eco en la jurisprudencia constitucional colombiana, como justicia material.

\section{Rawls en la Corte Constitucional colombiana}

En Colombia son numerosos los escritos filosóficos que abordan la teoría de Rawls bien sea para explicarla o para establecer vínculos con autores tan variados como Kant o Marx. Sin embargo, en el campo del derecho, ha sido más bien timorato el camino recorrido, lo que se evidencia en la escasa citación tanto en publicaciones jurídicas como en la jurisprudencia de las altas Cortes.

Tan solo una mirada a la base de datos de la Corte Constitucional colombiana permite rastrear citas de Rawls en 43 sentencias y un auto ${ }^{8}$ producidos entre 1992 y 2019. ${ }^{9}$ No obstante, muchas de esas citas son incidentales o de otras sentencias (es decir, reiteración de un fallo en donde previamente se lo había citado) o en salvamentos o aclaraciones de voto.

Como punto de partida para un futuro análisis sobre la incidencia de Rawls en la Corte Constitucional pueden establecerse la siguiente periodización y distinguirse los siguientes magistrados que citaron al filósofo norteamericano: a) entre los años 1992 a 1997 quienes citaron al filósofo fueron Ciro Angarita Barón, Jorge Arango Mejía, Carlos Gaviria Díaz y Alejandro Martínez Caballero; b) entre los años 2001 y 2008 lo citaron Rodrigo Uprimny, Clara Inés Vargas, Alfredo Beltrán Sierra, Jaime Araujo Rentería y Humberto Sierra Porto, y c) entre los años 2012 y 2018 se destacan María Victoria Calle, Adriana Mejía Guillen, Gloria

8 T-406/92; T-139/93; C-179/94; C-221/94; T-401/94; C-562/96; C-510/97; T-1207/01 (citado en la aclaración de voto); C-1260/01; C-893/01; C-654/04; C-668/04; C-338/06; SU-174/07; C-933/07; T-571/08; C-713/08; C-889/12 (citado en el salvamento de voto); T-320/12; SU-399/12; SU-400/12 (citado en el salvamento de voto); C-742/12; T-603/12; C-288/12; C-132/12; C-249/12; T-933/13; C-283/14 (citado en la aclaración de voto); C-387/14 T-541/14; C-284/15 (citado en la aclaración de voto); C-448/15 (citado en la aclaración de voto); T-097/16; C-205/16; C-209/16 (citado en el salvamento de voto); C-467/16 (citado en el salvamento de voto); T-507/16; C-699/16 (citado en el salvamento de voto); C-565/17 (citado en el salvamento de voto); C-041/17 (citado en el salvamento de voto); C-002/2018 (citado en el salvamento de voto); A-320/18 (citado en el salvamento de voto); C-045/19 (citado en la aclaración de voto).

9 En lo que va del año 2020, y hasta la fecha de redacción de este artículo, Rawls no ha sido citado en ninguna providencia de la Corte Constitucional. 
Stella Ortiz, Gabriel Eduardo Mendoza, Jorge Iván Palacio, Alberto Rojas Ríos y Luis Ernesto Vargas Silva.

Dicho lo anterior, se debe señalar que algunas de las características del rol del juez, expuestas en el acápite anterior, han sido adoptadas por la Corte Constitucional, quizás no por influencia directa de Rawls, sino más bien del sistema de precedentes norteamericano. Es así que la Corte, de tiempo atrás, ha estimado que debía aplicarse dicho sistema de precedentes para respetar el derecho a la igualdad en la medida en que a un caso similar cabría predicarse una respuesta similar a la adoptada en precedencia. Así, por ejemplo, en la sentencia de constitucionalidad C-836 de 2001, la Corte Constitucional sostuvo que

el principio de igualdad consagrado en el preámbulo de la Carta, en armonía con las diversas manifestaciones constitucionales de la igualdad -como derecho- tienen como presupuestos la igualdad frente a la ley, y la igualdad de protección y trato por parte de las autoridades del Estado.

También, en la Sentencia C-037 de 1996, la Corte dijo que la "independencia judicial tiene que armonizarse con el principio de igualdad en la aplicación del derecho". Es decir, el establecimiento de precedentes en la jurisprudencia se sustenta en el principio de igualdad que impide un trato desigual por parte de los jueces frente a una misma situación de hecho, lo que permite considerar la existencia de un sistema claro de reglas jurisprudenciales y una regularidad en la aplicación e interpretación del derecho.

De otra parte, al igual que Rawls destaca que a pesar del sistema de precedentes los jueces pueden apartarse de casos anteriores, previa justificación, la doctrina constitucional ha consagrado la misma posibilidad cuando se advierta que el caso en concreto presenta elementos diferenciadores con los anteriores (disanalogía), evento en el cual se le exige al juzgador una carga argumentativa que explique el porqué del tratamiento diferente. Al respecto, en la sentencia de tutela T-123 de 1995 se dijo que "se vulnera el principio de igualdad si se otorga un trato desigual a quienes se hallan en la misma situación, sin que 
medie una justificación objetiva y razonable", lo que constituiría una manifestación del principio de diferencia.

Así mismo, es factible ver cómo la teoría de los principios de la justicia orientada a la defensa de los derechos de las personas en posición menos ventajosa ha tenido cierta recepción en la jurisprudencia constitucional. En este orden de ideas, estimamos importante destacar la primera providencia de la Corte Constitucional que citó la obra de Rawls: la sentencia de tutela T-406 de 1992. Dicha sentencia es un hito en la historia del derecho porque puso a los abogados a pensar en las diferencias entre valores, principios y reglas de cara a los derechos fundamentales. Ciro Angarita Barón, abogado de la Universidad Nacional y ponente de la sentencia, citó Teoría de la justicia para introducir el tema de la justicia distributiva. Allí se dijo que el juez de tutela, al momento de decidir sobre la distribución de recursos públicos, debería "recurrir al principio de igualdad, ampliamente debatido en la teoría de la justicia de las últimas décadas, a partir del cual toda distribución de recursos, para ser justa, debe mejorar al menos la condición de los más desfavorecidos".

De igual manera, es copiosa la jurisprudencia constitucional que se orienta a amparar a las personas con algún tipo de merma física o mental, o también la que protege a personas en estado de indefensión como los menores de edad y los adultos mayores. Entre estos casos es importante destacar la línea jurisprudencial que existe en materia de protección de niños hiperactivos, pues en ella también se materializa el principio de igualdad. Así, por ejemplo, en la Sentencia T-255 de 2001, frente a un caso de hiperactividad infantil, trastorno que puede derivar en la estigmatización, depresión o angustia de los menores, dijo la Corte Constitucional que las situaciones concretas deben analizarse "desde la óptica del bien común y de la solidaridad", por ello:

No sobra señalar que a la luz del principio de igualdad, soporte fundamental del concepto de Estado Social de Derecho, se impone una acción de las autoridades y de la sociedad que no ha de ser siempre neutra, y ello con el fin de lograr el equilibrio necesario en pro de un sistema más justo y equitativo, fundado en la dignidad humana. 
De esta forma, se espera, por el contrario, que se otorgue un trato especial a los grupos sociales que se hallan en condiciones reales de indefensión o de inferioridad (Sentencia T-255 de 2001).

En otras oportunidades, la Corte Constitucional ha aplicado la llamada discriminación positiva como forma de acción afirmativa que se dirige a establecer un trato diferente para materializar la igualdad real de los menos favorecidos, como, por ejemplo, la Sentencia C-115 de 2017 en la que se analizó la constitucionalidad parcial de la Ley 1429 de 2010, sobre generación de empleos a jóvenes menores de 28 años. Allí, la Corte destacó como uno de los pilares fundamentales del Estado social de derecho la superación de la igualdad formal ante la ley para dar paso a la igualdad material que

se materializa en una regla de trato diferenciado o adaptado y de formulación de políticas públicas focalizadas, destinadas a corregir desigualdades de hecho de aquellas personas que por su condición económica, física o mental se encuentren en circunstancias de inferioridad, así como respecto de los grupos tradicionalmente marginados o discriminados. De esta manera, las políticas públicas, incluida la legislativa, cuando consultan el principio de igualdad en pro de lograr la igualdad material, realizan una actividad estatal que se califica como equitativa (Sentencia C-115 de 2017).

De las anteriores citas, se puede colegir que el derecho a la igualdad es el punto argumentativo que le permite a la Corte establecer un trato desigual en favor de una población en condición de especial protección $y$, si se quiere, menos favorecida. De esta manera, en la cuarta etapa del descorrimiento del velo de ignorancia se materializan los principios de la justicia, pero sobre todo el segundo principio, en casos particulares, lo que desde la perspectiva de la estructura del Estado permite insistir en la denominación de "etapa judicial" a este estadio de la teoría rawlsiana.

Se observa, entonces, que el juez constitucional termina aplicando los principios de la justicia expuestos por Rawls y adopta decisiones para garantizar la igualdad de oportunidades para los menos favorecidos. 


\section{Conclusión}

La obra de Rawls, para los momentos en que vivimos, resulta atractiva al menos por dos razones: por un lado, provee de una justificación racional al conocimiento jurídico necesaria para una época exageradamente rica en producción de normas y con una caótica jurisprudencia; por otro lado, plantea unos principios de la justicia que resultan necesarios si se quiere un nuevo pacto social, como se reclama desde cierto sector social en la actualidad.

La Teoría de la justicia de John Rawls es un texto que, a pesar de haber sido publicado en 1971, mantiene una vigencia y actualidad que se evidencia tanto en los debates académicos, como en la jurisprudencia colombiana, al menos la constitucional, porque Rawls no se queda en el contrato social original, sino que establece un procedimiento de aplicación de los principios de la justicia a todo el aparato estatal.

Las hipótesis de la posición original y del velo de ignorancia permiten describir los fenómenos que en derecho se denominan el "poder constituyente primario" y la gestación de una constitución en una "asamblea constituyente". Los dos principios expuestos por Rawls se han de plasmar en un texto constitucional y sirven como norte interpretativo de las actuaciones del Estado, incluso, del examen que a casos particulares realizan los jueces.

El primer principio, consagrado a las libertades y a los bienes básicos, resulta fundamental para la existencia misma de un Estado bien ordenado; empero, el segundo principio, sobre la igualdad y la protección de personas en situaciones menos ventajosas, adquiere especial relevancia de cara a la actuación de los jueces quienes resuelven conflictos concretos. En particular, los jueces constitucionales no solo por vía de control constitucional de las leyes, sino cuando actúan en sede de tutela confrontan los casos particulares con la estructura constitucional para materializar la anhelada igualdad material.

También se puede concluir que la Corte Constitucional colombiana de manera expresa ha acogido la teoría de la justicia de John Rawls en un número significativo de fallos. Además, muchas de sus consideraciones pueden apreciarse en la jurisprudencia constitucional así no 
obedezcan a una cita directa de la teoría rawlsiana. En los casos aquí citados, también se puede evidenciar como cierta la premisa de Rawls según la cual el principio de igualdad se materializa, o por lo menos se hace más evidente, en la actuación de los jueces, lo que constituiría la última etapa en la secuencia de aplicación de los principios de justicia (Rawls, 2011, p. 188).

Por último, se puede afirmar que Rawls ha contribuido de forma activa al debate universal sobre la justicia y ha enriquecido nuestras discusiones locales sobre la materia. Parafraseando a Robert Nozick (2017), no solo filósofos y políticos, sino también los abogados deben entablar un diálogo con la obra de Rawls bien para seguirla o explicar por qué no se ha de trabajar con ella. Con este texto se quiere despertar un mayor interés por los textos del filósofo norteamericano ya que, al menos en el campo del derecho, proveen una teoría clara y verificable para la estructuración de un Estado bien ordenado.

\section{Referencias}

Benjamin, W. (2010). "Hacia la crítica de la violencia”. En (J. Navarro, trad.), Obras II, tomo 1 (pp. 183-206). Editorial Abada.

Colombia, Corte Constitucional. Sentencia T-406 de 1992.

Colombia, Corte Constitucional. Sentencia T-123 de 1995.

Colombia, Corte Constitucional. Sentencia C-037 de 1996.

Colombia, Corte Constitucional. Sentencia T-255 de 2001.

Colombia, Corte Constitucional. Sentencia C-836 de 2001.

Colombia, Corte Constitucional. Sentencia C-551 de 2003.

Colombia, Corte Constitucional. Sentencia C-115 de 2017.

Derrida, J. (2010). Fuerza de Ley. El «fundamento místico de la autoridad» (A. Barberá \& P. Peñalver, trads.). Editorial Tecnos.

Dworkin, R. (2014). Justicia para erizos (H. Pons, trad.). Fondo de Cultura Económica.

Edmundson, W. (2017). John Rawls: Reticent socialist. Cambridge University Press.

Freeman, S. (2016). Rawls (A. García de la Siena, trad.). Fondo de Cultura Económica. 
García Jaramillo, L. (2004). La revitalización en el modo de hacer filosofía política por la Justicia como Equidad de John Rawls. Cuadernos FilosóficoLiterarios, (15), 97-128.

Grueso, D. (2005). “John Rawls; Legado de un pensamiento". En Autor, John Rawls. Legado de un pensamiento (pp. 11-29). Universidad del Valle.

Hegel, G.W.F. (1979). Lecciones sobre la historia de la filosofía, vol. III (W. Roces, trad.). Fondo de Cultura Económica.

Jaramillo, J. F., García, M., Rodríguez, A. A., E Uprimmy, R. (2018). El derecho frente al poder. Surgimiento, desarrollo y crítica del constitucionalismo moderno. Universidad Nacional.

Kant, I. (2008). La metafísica de las costumbres (A. Cortina E J. Conill, trads.). Editorial Tecnos.

Kennedy, D. (2010). “El comportamiento estratégico en la interpretación jurídica". En (G. Moro, trad.), Izquierda y derecho. Ensayos de teoría jurídica crítica (pp. 27-83). Siglo XXI Editores.

Mancha, P. (2009). "La Justicia como Equidad y el constitucionalismo popular. Aproximación al sujeto normativo en John Rawls y Larry Kramer". Revista de Derecho UNED, (5), 253-286. https://doi.org/10.5944/rduned.5.2009.10987

Martínez, D. (2015). “Una aproximación al pensamiento económico-normativo de John Rawls". Sociedad y Economía, (28), 95-112. http://www.redalyc. org/articulo.oa?id=99634857007

Nagel, T. (2003). "La compasión rigurosa de John Rawls: una breve biografía intelectual" (P. Arango y L. García Jaramillo, trads.). Revista Praxis Filosófica, 25-40. https://bibliotecadigital.univalle.edu.co/bitstream/ handle/10893/1859/Art\%20002\%20La\%20compasion\%20rigurosa\%20 de\%20John\%20Rawls.pdf?sequence $=1$ EisAllowed $=\mathrm{y}$

Nozick, R. (2017). Anarquía, Estado y utopía (R. Tamayo, trad.). Fondo de Cultura Económica.

Nussbaum, M. (2004). "Hacer que la filosofía se ocupe de la política" (L. García, trad.). Cuadernos Filosófico-Literarios, (16-17), 23-26.

Puyol González, A. (2016). Rawls. El filósofo de la justicia. Emse Edapp S.L.

Rawls, J. (2011). Teoría de la justicia (M. Dolores, trad.). Fondo de Cultura Económica.

Rawls, J. (2012). Justicia como equidad: una reformulación (A. de Francisco, trad.). Paidós.

Rawls, J. (2015). Liberalismo político (S. R. Madero, trad.). Fondo de Cultura Económica. 
Rico S., R. (2005). Genealogía del concepto de poder constituyente primario en Colombia. (Trabajo de grado, Pontificia Universidad Javeriana, Bogotá, Colombia).

Spinoza, B. (2008). Tratado teológico-político (A. Dominguez, trad.). Alianza Editorial. 